\section{RE-PENSANDO EL USO DE LAS TIC EN EDUCACIÓN: REFLEXIONES DIDÁCTICAS DEL USO DE LA WEB 2.0 EN EL AULA ESCOLAR}

\author{
Marcelo Arancibia Herrera \\ marancibiah@gmail.com \\ Luis Cárcamo Ulloa \\ luis.carcamo@gmail.com \\ Paulo Contreras Contreras \\ contrerasuach@gmail.com \\ Eliana Scheihing García \\ eliana.scheihing@gmail.com \\ Daniela Troncoso Vargas \\ danielatroncoso.87@gmail.com
}

Universidad Austral de Chile Valdivia - Chile

Cómo citar este artículo/Citation: Arancibia Herrera, M.; Cárcamo Ulloa, L.; Contreras Contreras, P.; Scheihing García, E. y Troncoso Vargas, D. (2014). "Re-pensando el uso de las TIC en educación: reflexiones didácticas del uso de la Web 2.0 en el aula escolar". Arbor, 190 (766): a122. doi: http://dx.doi. org/10.3989/arbor.2014.766n2014

Recibido: 4 marzo 2013. Aceptado: 16 enero 2014.

RESUMEN: Desde 1996 el Estado chileno ha desarrollado una política de incorporación progresiva de tecnologías al aula. Al mismo tiempo los jóvenes usan cada vez de forma más intensiva las TIC y en particular los servicios de la Web 2.0. Sin embargo, hay evidencia que el uso de las tecnologías desde una perspectiva didáctica y creativa no es habitual. La propuesta didáctico-pedagógica de Kelluwen busca articular el trabajo de aula con herramientas de la Web 2.0 a través del uso de Diseños Didácticos Colaborativos para desarrollar competencias sociocomunicativas y contenidos disciplinares en los estudiantes. Este artículo sistematiza, expone y discute los resultados preliminares de una intervención educativa que ha consolidado una comunidad de estudiantes, profesores e investigadores centrada en construir, usar y compartir diseños didácticos colaborativos que utilizan la Web Social como espacio de mediación en el proceso de enseñanza y aprendizaje escolar.

PALABRAS CLAVE: Contextos escolares; experiencias didácticas; aprendizaje mediado por TIC; aprendizaje colaborativo; competencias comunicativas.

\section{RETHINKING THE USE OF ICT IN EDUCATION: DIDACTIC REFLECTIONS OF THE USE OF WEB 2.0 IN THE CLASSROOM}

Copyright: (C) 2014 CSIC. Este es un artículo de acceso abierto distribuido bajo los términos de la licencia Creative Commons Attribution-Non Commercial (by-nc) Spain 3.0.
ABSTRACT: Since 1996, the Chilean government has progressively incorporated technology into almost every classroom. This adds to the finding that young people are increasingly using ICT and services including Web 2.0 more intensively. However, there is evidence that the use of ICT from a creative and educational perspective is uncommon. The "Kelluwen" didactic-pedagogic proposal seeks to articulate classroom-work to innovate using Web 2.0 tools, through the use of Collaborative Education Design in order to develop socio-communicative skills in students from 7 th to 10 th grade (Basic and Middle School). The paper summarises the results of three years' work setting up and consolidating a community of students, teachers and researchers focused on building, using and sharing didactic experiences.

KEYWORDS: School contexts; didactical experiences; ICT'S mediated learning; collaborative learning; communicative skills. 


\section{USO DE TIC EN EL CONTEXTO EDUCACIONAL CHILENO: LA DIDÁCTICA COMO TAREA PENDIENTE}

En concordancia con los procesos de tecnologización de la vida cotidiana, resulta poco probable que las instituciones educativas sean impermeables a los cambios socio-tecnológicos (Bonder, 2008; Chinn y Fairlie, 2010). En este contexto parece obvio que la Escuela viva un proceso de re-conceptualización no sólo estructural, sino también de sentidos, lo que se expresa en una creciente desescolarización de la enseñanza, la pérdida de protagonismo del profesor y la difuminación de las fronteras disciplinares en el currículo.

Así pues, estas transformaciones sociales paradigmáticas podrían implicar un nuevo modo de conocer y, por tanto, de aprender. En educación, el constructivismo social hace ya tiempo, gana adeptos sobre un aprendizaje memorístico (Gardner, 1993; Monereo, 2005). En consecuencia, en los nuevos tiempos la educación formal como institución ha perdido la tutoría exclusiva del proceso de enseñanza aprendizaje. El ordenador e Internet en los hogares permiten que las personas tengan la información en sus casas, por lo que el acceso al conocimiento deja de ser un rol hegemónico de la escuela. Desde allí, resulta importante saber qué hacer o cómo utilizar la gran cantidad de contenidos que circulan en los medios y recursos que dispone la escuela y, por lo mismo, se ha de poner mayor atención en los procesos didácticos sobre los cuales se instalan las Tecnologías de la Información y la Comunicación (en adelante TIC). Es así como se ha de transitar de un tipo de uso instrumental de las TIC por parte del profesorado hacia usos creativos de los ambientes que hoy están en la red (Prain y Hand, 2003), en particular la Web 2.0, para que los estudiantes aprendan cuestiones relacionadas a las competencias necesarias para el mundo digital.

Es importante señalar que la investigación educativa reconoce el rol fundamental que juegan los profesores en la renovación educacional, al tiempo que plantea la importancia de su papel en la incorporación curricular de las TIC (Meier, 2005; Zhao y Frank, 2006). Asimismo, estudios comparados han demostrado que existe la convicción de parte de los profesores que la incorporación de las TIC en la educación es un elemento positivo (SITES, 2006). No obstante, a pesar de dicha convicción y del aumento de la cantidad de ordenadores con conexiones a Internet en las escuelas, las investigaciones revelan que su uso didáctico y creativo no es tan habitual y tiene dificultades (Crook, 2012; García-Valcárcel y Tejedor, 2010; Hillis y Munro, 2005; Sigalès, Mominó, Meneses y Badia, 2009,).
Por otro lado, el particular panorama escolar chileno actual es heterogéneo y los establecimientos educativos dan cuenta de la estructura social desigual, por medio de una pertenencia estructural disímil de colegios públicos, particulares concertados y particulares pagados, donde podemos ver ostensibles brechas entre los colegios que pertenecen a los estratos socioeconómicos medio-alto y alto, y los que pertenecen al grupo medio-bajo y bajo (Arancibia y Contreras, 2013). Esto se explica, en cierto modo, por las reformas impulsadas en los años ' 80 por la dictadura militar que modifica el sistema escolar, transitando desde un Estado Docente a uno Subsidiario, descentralizando la administración económica a nivel de municipios, pero manteniendo centralizadamente en el ministerio de educación aspectos como el currículum y la evaluación pedagógica (Beyer, 2000; Cox, 2005).

En esta particular situación, desde 1996 el Estado chileno ha llevado una política de incorporación de nuevas tecnologías en el aula a partir de la implementación piloto en la Región Metropolitana del Proyecto ENLACES, programa que intervino en el desarrollo profesional docente, así como también cooperó en la modernización del sistema escolar, impactando a la totalidad de la comunidad educativa (Arancibia, 2000). Así es como, se considera que el Proyecto ENLACES fue un aporte a la reducción de la brecha digital de las nuevas generaciones resolviendo en parte esta diferencia a través de planes de cobertura tecnológica y capacitación docente (Hepp, 2003).

Dada esta instalación tecnológica en las escuelas y liceos, avanzando hacia una perspectiva de intervención, que permita re-pensar la integración de las TIC (Staples, et.al., 2005) es legítimo preguntarse ¿Cómo se puede resolver la brecha que existe entre las didácticas anquilosadas y el uso de TIC en contextos escolares?, ¿Cómo es posible acercar las actividades escolares de aprendizaje a los usos de la web social que hacen los estudiantes?

A continuación presentamos una iniciativa realizada en Chile durante tres años, intercalando en todo momento una discusión teórica que da cuenta de los sustentos y fundamentos que están a la base de cada decisión didáctica tomada. Por tanto, pretendemos que no sea una exhibición instrumental de una secuencia instruccional, al modo de receta para obtener aprendizajes superficiales.

\section{LA EXPERIENCIA KELLUWEN ${ }^{1}$}

La propuesta de Kelluwen busca responder al núcleo de la problemática enunciada, a través de la im- 
plementación de Diseños Didácticos Colaborativos (en adelante DDC) con uso de recursos de la Web 2.0. Se trata de una iniciativa que entremezcla elementos de aprendizaje basado en proyectos y aprendizaje colaborativo (Palacheewa, Suwannatthachote \& Nilsook, 2012). La propuesta didáctico-pedagógica de Kelluwen busca potenciar el trabajo de aula para profesores interesados en innovar con herramientas de la web 2.0 o Web Social. Las intervenciones se ejecutan apoyadas por la plataforma que también lleva el nombre del proyecto, a la cual se accede vía http://www.kelluwen.cl

En este espacio profesores y profesoras pueden encontrar 16 DDC que trabajan contenidos curriculares regulares de 7ㅇ básico a 4을 medio (últimos 6 años de enseñanza obligatoria en Chile) para las asignaturas de Lenguaje y Comunicación e Historia, Geografía y Ciencias Sociales. Si bien los contenidos resultan bastante apegados a los planes y programas propuestos por el Ministerio de Educación de Chile, la intención del equipo de investigación fue incorporar un uso creativo y dialógico de los recursos de la web social. Es por ello que cada diseño se apropia de una aplicación de la web social, tal como Youtube, Wordpress, Panoramio o Gigapan, entre otros, para convertir a estas herramientas en parte activa del escenario pedagógico en las aulas de los jóvenes.

Kelluwen está pensado como una propuesta de Blearning (Bartolomé, 2004), pues la coordinación del proceso está a cargo del profesor responsable de la asignatura. En tal sentido Kelluwen no es educación a distancia vía Internet, sino un proceso que busca revitalizar el aula de colegios vulnerables de Chile. También entendemos que el B-learning se hace parte del proceso en tanto la propuesta busca el equilibrio entre la clases en el laboratorio de informática educativa y el aula regular, ya que se intercalan sesiones en ambos espacios, de modo que la tecnología sea un aporte real al desarrollo de una pedagogía activa y no un distractor que dificulte el manejo de los grupos de estudiantes.

La colaboración y la aproximación a una mayor horizontalidad de los roles Profesor/Alumno intenta ser la columna vertebral del modelo. Desde la primera actividad se busca modelar el trabajo pedagógico en base de los conocimientos previos y las opiniones existentes en el aula, relacionándoles a los contenidos curriculares que se abordarán durante la innovación. La colaboración, por su parte, se establece en dos espacios: a)el desarrollo de actividades o proyectos grupales en que participan de 3 a 5 estudiantes, y b)la relación con un aula gemela que estará ejecutando el mismo diseño didáctico en otro punto geográfico del país. El aula gemela valora y opina sobre las evidencias de aprendizaje creadas por otros estudiantes provenientes de otros contextos escolares. Todo lo anterior está guiado por pautas que la plataforma provee, y coordinado gracias a un mecanismo de programación informática que va siguiendo los estados de avance de los grupos y asignando pares revisores que se hallan en el mismo estadio de progreso de las actividades, pero no necesariamente en la misma aula. De esta forma, un grupo de un aula de la ciudad de Valdivia le correspondió revisar, comentar y valorar el trabajo de otros grupos de localidades rurales o distantes geográficamente, como Futrono o Hualaihué.

Por otra parte, consideramos que el acto de enseñanza aprendizaje es también un acto dialógico de comunicación (Aubert, Flecha, García y Racionero, 2011) en el que intervienen actores dentro y fuera del aula. Cuando un niño o un adolescente ejecutan una tarea en la sala de clases está interactuando directamente con su profesor y algunos de sus compañeros; y además el producto de esa tarea o evidencia de aprendizaje específica será un mensaje que -más allá del esfuerzo intelectual- busca ser comunicado a su comunidad de estudio. Si todo acto de aprendizaje es un acto de comunicación, no es difícil suponer que sin importar el contenido curricular en juego, el lenguaje es el articulador de cualquier escena didáctica. Resulta necesario comprender que en la escuela se requiere más que una alfabetización digital funcional (Arancibia, 2007). En la actualidad parece indispensable desarrollar competencias comunicativas, entendidas como habilidades que permitan a los individuos asumir compromisos de interacción, considerar normas elementales contextuales para establecer relaciones con otros y la comprensión de los diferentes signos, significados y códigos presentes en los con-textos socioculturales. Dichas habilidades siguen hoy enseñándose en el aula como si fuesen un simple proceso de transferencia de información, prescindiendo totalmente del texto situado en un con-texto, se ignora si los procesos mentales que el sujeto pone en juego son los adecuados. En una mirada actualizadora Coll (2005) considera que el hipertexto y la Web vienen a potenciar nuevas habilidades en lo referente a comprensión lectora y producción de texto.

\section{LA PROPUESTA DIDÁCTICA}

La propuesta didáctica y metodológica de Kelluwen corresponde a un DDC estructurado en tres etapas 
que contienen objetivos curriculares y evidencias de aprendizaje esperadas que, a su vez, forman en conjunto un aprendizaje continuo, una construcción del conocimiento sustentada en procesos de cognición y metacognición que buscan desarrollar transversalmente la criticidad en el proceso de enseñanzaaprendizaje.

Algunos de los elementos que configuran la experiencia didáctica corresponden a ejercicios articulados a través de un modelado del diseño didáctico en acciones en las que el estudiante debe autorregular su proceso de aprendizaje (Álvarez, 2009). Cada DDC dura entre 4 y 6 semanas de clases. Los aspectos del modelado pedagógico de cada diseño comprenden macro etapas de motivación, creación y evaluación, que detallaremos a continuación y que se representan en la figura 1.

\section{a) Etapa de Motivación}

Esta primera etapa implica la socialización de conocimientos previos de los estudiantes respecto de los objetivos curriculares y transversales a desarrollar. La motivación se realiza en 2 o 3 sesiones en las cuales el profesor da a conocer a los estudiantes las características generales del DDC, la metodología de trabajo a partir de grupos colaborativos, la organización del proceso de enseñanza-aprendizaje en cada una de sus etapas y la construcción de conocimiento mediante actividades que persiguen evidencias de aprendizaje que incorporan herramientas de la Web 2.0. En esta fase se requiere explicar a los estudiantes la distribución de los momentos de cada sesión y los roles que cada uno desempeñará durante la experiencia didáctica. El docente como un guía y colaborador de los procesos de aprendizaje recupera experiencias previas y presenta los contenidos a partir de los contextos desde los que cada estudiante coopera y participa.

A partir de estos diálogos en el aula cada docente propicia estrategias desafiantes, estructuradas y significativas para los estudiantes, tal como lo expone el Marco para la Buena Enseñanza establecido en Chile por el Ministerio de Educación "Propone actividades que involucran cognitivamente a los estudiantes y entrega tareas que los comprometen en la exploración de contenidos" (MINEDUC, 2003:11). El compromiso de los estudiantes en el proceso de enseñanza-aprendizaje es fundamental para garantizar un desarrollo efectivo de las evidencias de aprendizaje (recopilación de información, edición y producción de evidencias) y también para generar una evaluación auténtica que esté dada por la observación constante del profesor y la atención de cada estudiante ante la construcción de conocimientos derivados de los procesos individuales y colectivos ejecutados a lo largo de la experiencia didáctica (Álvarez, 2009).

Dado que los DDC son propuestas de trabajo colaborativo que persiguen un sentido de entreaprendizaje (Prieto, 1996), será fundamental lograr el compromiso por parte de los estudiantes, situación que no es difícil de estimular si se explica el valor de la

Figura 1. Esquema de estructuración de Diseños Didácticos Colaborativos (DCC)

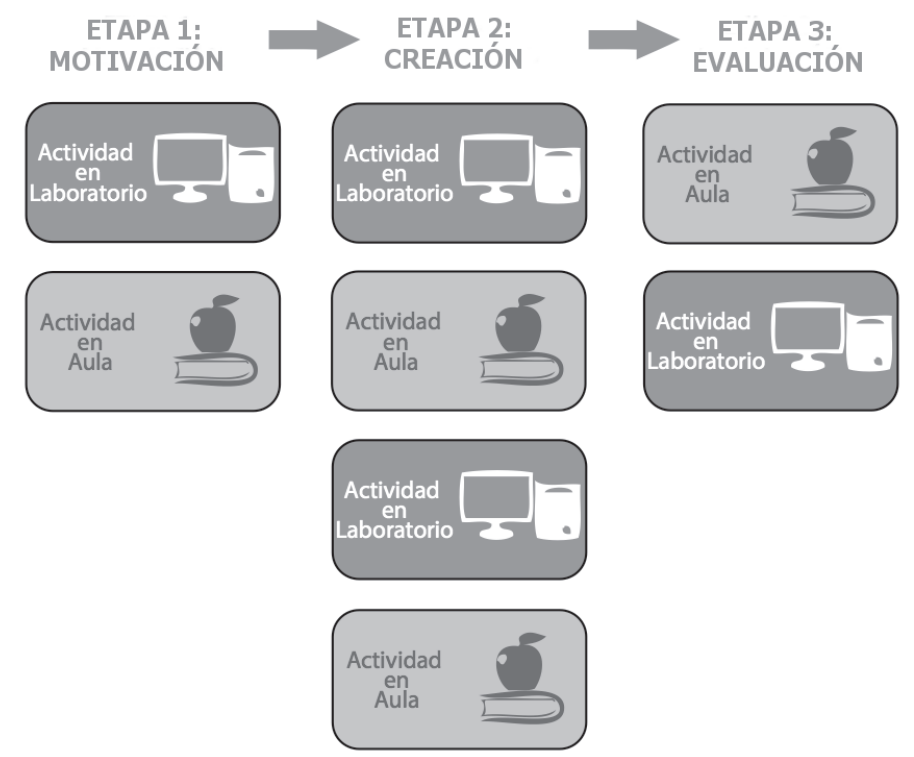


innovación y la utilización de Internet como soporte social con un sentido comunicativo.

A cada integrante del grupo le es asignado un rol, esta responsabilidad permite la apropiación del proceso de aprendizaje en la medida que el estudiante, al cumplir un papel relevante dentro de la experiencia didáctica, se hace parte del proceso desde una perspectiva participativa. De este modo, cada estudiante pasa a integrar una pieza del engranaje que articula el trabajo en equipo, tanto para la construcción de la evidencia de aprendizaje, como para la evaluación clase a clase de las actividades que se desarrollan. A continuación se exhibe un ejemplo de distribución grupal propuesto para el DDC de historia "Un diaporama del siglo XX":

- Coordinador equipo: quien es responsable del contenido y su publicación, se encarga de hacer que el grupo funcione y se cumplan las tareas. Perfil: estudiante con liderazgo reconocido.

- Informático: responsable de organizar la cuenta en Youtube y publicar los trabajos en la plataforma kelluwen, es decir el responsable de los elementos informáticos operativos (cuentas, contraseñas, ingresos, categorías, etc.). Perfil: El “informático” del grupo.

- Investigador: responsable de conseguir información adecuada para publicar. Perfil: aquí se puede asignar al más "aislado" o "individualista" del grupo pues puede buscar información por su cuenta y luego la comparte.

- Historiador: responsable de cautelar la "veracidad" y adecuación de la información encontrada. Perfil: estudiante que demuestra interés y/o un alto rendimiento en la asignatura. El historiador también se encarga del aseguramiento de calidad, por ejemplo: respetando el referenciar la información extraída de otras fuentes.

\section{- Apoyador: miembro que participa transversal- mente de todas las funciones, es quien posibi- lita el trabajo integrador y/o reemplaza en al- guna tarea en caso de ausencia. Perfil: el más "desordenado" quien puede y tal vez requiere cambiar de rol constantemente.}

Esta perspectiva se cumple a medida que los estudiantes concretan las actividades propuestas en esta etapa y reconocen cómo las experiencias previas y percepciones acerca de los contenidos se integran al nuevo proceso de enseñanza-aprendizaje. Activi- dades como debates, mesas redondas, foros, diagnósticos de problemáticas en torno a un tema, entre otros, se presentan como oportunidades de generar momentos de dinamismo comunicativo en que los estudiantes experimentan ejercicios de opinión o reflexión, donde la propuesta curricular se relaciona con datos y opiniones que forman parte de su propia realidad. Dicha propuesta busca formar un ambiente estimulante que sea lo más favorable para quienes se encuentran en la sala de clases, propiciando un entorno basado en el respeto por el otro y la solidaridad del trabajo colaborativo, así se valoran los ejes propuestos por el profesor y las dudas, opiniones y creaciones que realizan los estudiantes (MINEDUC, 2003).

Como se ha mencionado con anterioridad, durante la etapa motivacional es primordial enfocar el proceso de enseñanza-aprendizaje rescatando los conocimientos previos de los estudiantes. Según Álvarez (2009) las tareas realistas o relevantes para los jóvenes se caracterizan por contener en su modelado un vínculo con el mundo real y cotidiano que permite una comprensión del aprendizaje desde la experiencia particular de cada individuo, asignando un valor afectivo y emocional que es esencial en la motivación por descubrir y aprender.

\section{b) Etapa de Creación}

Esta etapa está destinada a la elaboración de evidencias que confirmen el aprendizaje alcanzado. En ella se debe articular correctamente la inserción de los contenidos curriculares que cada diseño en particular busca cubrir y el desafío de construir una pedagogía activa apoyada por la Web 2.0. Los estudiantes se embarcan en un proceso de investigación y construcción colaborativa de conocimientos sobre temáticas o contenidos que están presentes en el currículo escolar hasta conseguir la elaboración de evidencias de aprendizajes que tomarán forma de mensajes audioescritovisuales (Cebrian, 2009; Cloutier, 2001), los cuales quedarán disponibles en la red.

Es importante que los estudiantes sepan que estas evidencias o avances serán, más tarde, auto y coevaluados por sus compañeros de clase y de las aulas gemelas, con el objetivo de revisar constantemente el trabajo que desarrollan y mejorar la calidad mediante la observación crítica del propio desempeño. Durante la etapa de creación el número de sesiones se encuentra determinado por la carga horaria que propone el currículum nacional para cada una de las áreas, abarcando de 5 a 7 sesiones el sector de Lenguaje y Comunicación, y de 4 a 5 sesiones el de Historia, Geografía y Ciencias Sociales. 
En la etapa de creación se propone una planificación orientada a que los grupos elaboren, paso a paso y de forma colaborativa, evidencias de aprendizaje que son publicadas en herramientas de la web 2.0.

Las primeras actividades están destinadas a buscar y seleccionar información desde la web, libros, cartas, biografías, realización de entrevistas, fotografías personales, familiares o del entorno, entre otros. Toda esta exploración de medios y actividades se orienta mediante guías y pautas de trabajo según el propósito didáctico y curricular de cada diseño y la herramienta web 2.0 asociada. Por ejemplo, en el DDC de lenguaje "Ha llegado Carta" los estudiante buscan cartas en medios de prensa digitales y las analizan mediante una guía que se ofrece como material de apoyo.

En cuanto al rol de los estudiantes, cada uno debe velar por la calidad de los mensajes elaborados según los roles asignados en la primera etapa de la experiencia didáctica, son todos responsables del trabajo colaborativo que crean y construyen en conjunto. Además, como ya se apuntó, todo el material que es recopilado se encuentra sujeto a una constante discusión y evaluación crítica por parte de los estudiantes, puesto que es publicado en las redes sociales. En este sentido, los DDC propician la autorregulación del proceso de aprendizaje, lo cual se posibilita mediante el tipo de tareas que se proponen en los DDC. Para cumplir este propósito los DDC ofrecen como material complementario una o dos pautas de auto y coevaluación para cada una de las actividades que se proponen.

Al finalizar la etapa de desarrollo cada grupo de trabajo habrá creado sus evidencias de aprendizajes como audiovisuales o textos multimodales para distintas plataformas. Con el apoyo de estas herramientas cada grupo de trabajo busca plasmar en su evidencia de aprendizaje la síntesis de la investigación realizada. Luego, las evidencias de cada grupo se comparten con la propia aula y con las aulas gemelas mediante su publicación en una herramienta Web 2.0 y la herramienta de gestión inteligente aportada por la plataforma Kelluwen en la sección de Trabajos, tal cual se muestra en un pantallazo de dicha sección en la figura 2 .

\section{c) Etapa de Evaluación}

En esta etapa se busca evaluar de modo integral la ejecución del DDC y los objetivos curriculares socializando los niveles de logro a través de la evaluación de evidencias de aprendizaje. Además, se busca hacer un barrido desde los conocimientos previos que iniciaron la experiencia innovadora hasta la construcción de nuevos conocimientos propuestos en el aula propia y por las gemelas.

Durante la etapa de evaluación, la organización de los tiempos se propone en función de actividades de síntesis a partir de las cuales los grupos de trabajo socializan y comparten la experiencia didáctica a través de la Herramienta de "Trabajos" que aporta la plataforma Kelluwen.

Esta herramienta es un agente inteligente que proporciona la plataforma donde las evidencias de aprendizaje realizadas por un grupo de estudiantes en un colegio son compartidas para su evaluación y valoración a un grupo de otro colegio que se encuentre en tiempos y ritmos similares. Para canalizar este trabajo se pro-

Figura 2. Vista de Herramienta de co-evaluación "Trabajos"

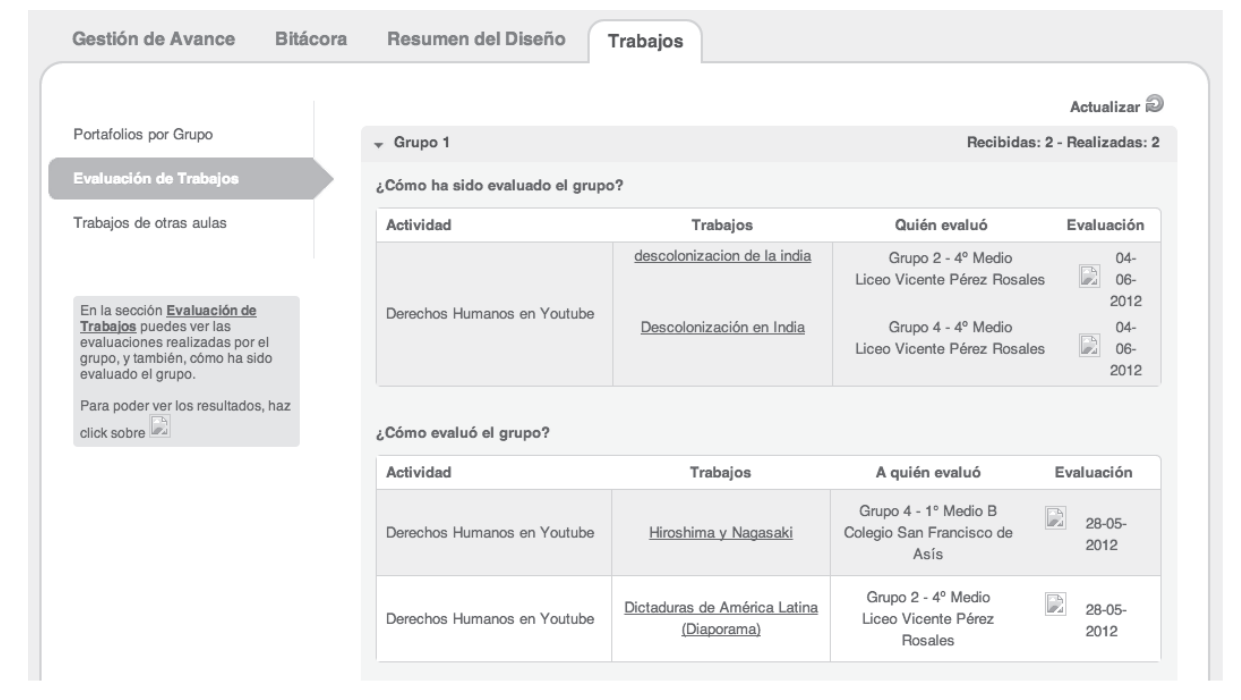


ponen actividades de evaluación en las que un grupo revisa y evalúa una evidencia de aprendizaje perteneciente a un aula gemela, para luego finalizar la experiencia didáctica realizando una autoevaluación grupal o individual orientada a reflexionar sobre los procesos metacognitivos desarrollados durante el aprendizaje.

Las evidencias de aprendizaje elaboradas por cada grupo son expuestas al curso en una sesión destinada a la observación y retroalimentación de los contenidos, para ello cada grupo presenta el producto final que han creado, junto con comentar las experiencias significativas durante el desarrollo de la investigación y creación de esta evidencia. El propósito de esta actividad es generar un momento en que los estudiantes reflexionen sobre el trabajo realizado durante la experiencia didáctica y valoren críticamente los resultados que han obtenido. Desde esta perspectiva, es fundamental que se generen preguntas, comentarios o discusiones por parte de todos los grupos que exponen, oportunidad en que cada estudiante ve reflejado su propio proceso de aprendizaje en las experiencias del otro.

Durante la etapa de evaluación está contemplada la interacción con aulas gemelas, la cual permite la visualización de diversas evidencias de aprendizaje. Esta es una gran posibilidad para que los estudiantes conozcan más de un enfoque u opinión respecto de un mismo objeto de estudio. Mediante este ejercicio los estudiantes reconocen que se pueden acceder a distintas opiniones y formas de generar modos explicativos variados para un contenido.

Cabe destacar que además de ser accesibles por medio de la plataforma Kelluwen las evidencias de aprendizaje quedan disponibles en las herramientas de Web Social que cada DDC propone, por ejemplo evidencias terminadas de aprendizaje publicadas en diferentes plataformas pueden visualizarse en: http:// www.youtube.com/watch?v=umrkOfiPJPw; http://issuu.com/marancibiah/docs/3282_choque_cultural_ terminado_; http://trab-infantil-grupo3-lichornop. blogspot.com/

Otra forma de acceder a los videos elaborados por los estudiantes es el playlist en Youtube: http://www. youtube.com/playlist?list=PL80BDA6FEA5385635

El proyecto tiene a la fecha 16 DDC a disposición de las comunidades escolares los cuales se describen a continuación en la tabla I para lenguaje y comunicación y en la tabla II para Historia y Ciencias Sociales:

Durante los 6 semestres en que se ejecutó el proyecto, se involucraron 57 centros escolares de $17 \mathrm{mu}$ nicipios diferentes de tres regiones del País. Se implementaron 161 experiencias didácticas con un total de 4517 estudiantes y 147 profesores. En relación al desarrollo de las experiencias podemos puntualizar que 33 de ellas alcanzaron un avance inferior al $70 \%$, otras 33 alcanzaron un avance entre 70 y $99 \%$, mientras que 95 lograron completar todas las actividades, es decir un $100 \%$ de avance.

Desde que se creó la Herramienta de Trabajos, en el segundo semestre del 2011, se subieron 320 trabajos, a partir de los cuales se generaron 640 vinculaciones para revisión de pares. De estas vinculaciones, se realizaron efectivamente 445 co-evaluaciones, y de ellas 405 estaban completas (rúbrica y comentario), siendo 268 (66\%) de ellas provenientes de grupos de aulas gemelas y 137 (34\%) de grupos de la misma aula.

Figura 3. Lista de reproducción con evidencias de aprendizajes y materiales divulgativos
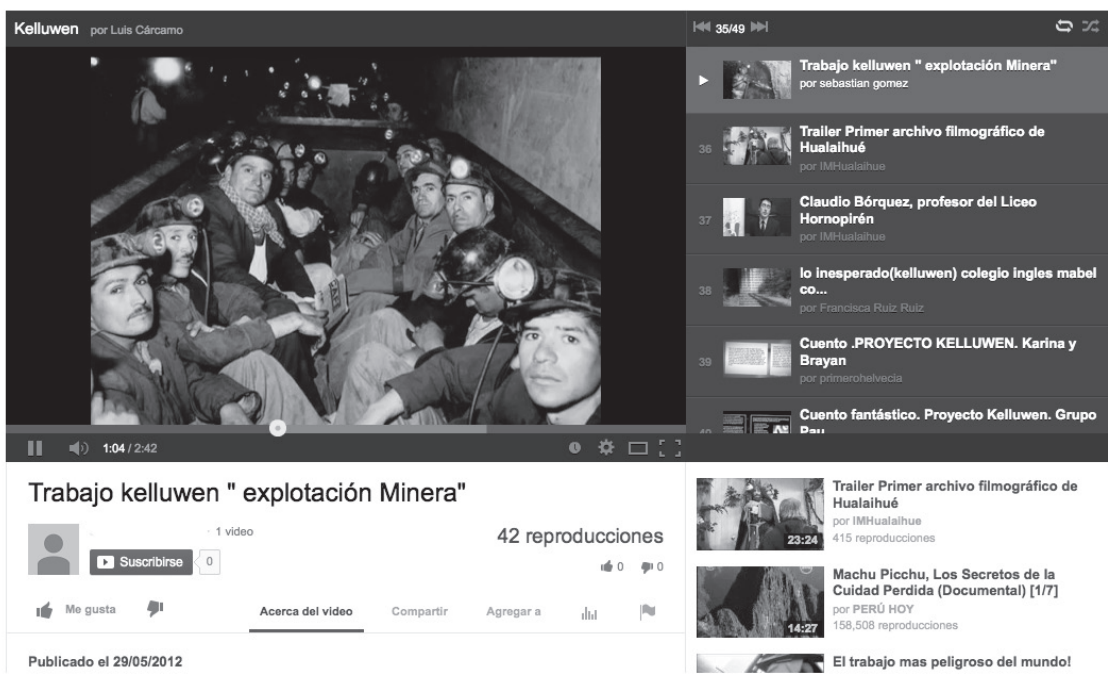
Tabla I. Resúmenes DDC creados y validados Lenguaje y Comunicación

1- El Blog Turístico de mi localidad. Posibilita la producción de un texto expositivo, con el objetivo de recomendar atractivos turísticos de su localidad. Se enfatiza en el proceso de escritura, en sus diferentes etapas, para afianzar la planificación y redacción de este tipo de textos empleando recursos gramaticales y léxicos. Una vez finalizada la experiencia didáctica, será posible apreciar, en el blog de cada aula, una serie de Informes que se constituirán en guía turística para quien desee revisar su contenido.

2- Te cuento mi cuento. Busca aplicar y profundizar elementos relacionados con el análisis de textos narrativos literarios. El diseño se propone trabajar un cuento a nivel grupal, distinguiendo diferentes momentos del proceso de comprensión lectora. A partir del análisis grupal del cuento, los grupos trabajarán utilizando GigaPan para compartir con otras aulas su texto literario. Para finalizar y conocer el trabajo realizado por los otros y evaluar el trabajo personal desarrollado se organizan actividades para retroalimentar la actividad.

3- Lo literario y lo no literario. El objetivo de este diseño didáctico es que los estudiantes lean, valoren y produzcan textos literarios y no literarios. El desarrollo de los aprendizajes requiere que los estudiantes elaboren a lo largo del diseño didáctico textos literarios y no literarios de manera grupal para publicarlos en Youtube, como diaporama o video, con el propósito de ser discutidos y comentados por los estudiantes del mismo curso y de las aulas gemelas.

4- Ha llegado Carta. Profundiza elementos de la comunicación verbal escrita, específicamente en dos tipos de textos: la biografía y la carta. Para ello los estudiantes, después de reconocer la estructura del texto biográfico, redactarán una autobiografía. Por medio de una carta de amistad compartirán con los estudiantes del aula gemela, sus autobiografías. Finalmente, los grupos de trabajo procederán a responder las cartas y a evaluar el Diseño Didáctico de acuerdo a los aprendizajes adquiridos y a su desempeño.

5- Blogeando opiniones construyendo realidades. Elaboración de opiniones escritas a partir de temas controversiales y de interés. Para ello revisarán distintas fuentes de información en la web (periódicos, revistas, canales de televisión, radio, etc.). Las y los estudiantes publicarán sus opiniones en un blog, espacio en el que también opinarán y comentarán las opiniones de grupos de aulas gemelas.

6- Corre video: Los mundos literarios. Tiene como propósito la producción de material audiovisual a partir del reconocimiento de tipos de mundo según la realidad que representan: Mundo cotidiano, Mundo onírico y Mundo mítico. Los estudiantes elaborarán un texto narrativo a partir del cual desarrollarán el material audiovisual, el que será publicado en la plataforma Youtube y compartido con los grupos gemelos.

7- El discurso del comentario en voicethread. El objetivo es la creación de una reseña escrita tomando como objeto de comentario un cortometraje. Para ello, los alumnos utilizan herramientas como procesadores de texto o presentaciones de diapositivas. Al mismo tiempo, el diseño contempla comentar la producción entre compañeros del aula y aulas gemelas a través de la plataforma VoiceThread. El contenido articulatorio de este diseño es el del discurso expositivo.

8- Notiblog. El propósito es que los estudiantes reconozcan las funciones referencial, apelativa y expresiva del lenguaje a través de la producción, análisis e interpretación de algunos textos periodísticos, principalmente la noticia y la entrevista. El progreso y evaluación del aprendizaje se realiza a través de la construcción de un blog, donde los estudiantes publican sus trabajos y actividades en un formato similar al periódico. Al finalizar la segunda etapa los estudiantes podrán compartir su trabajo con sus aulas gemelas para evaluarse mutuamente.

Tabla II. Resúmenes DDC creados y validados Historia y Ciencias Sociales

\begin{tabular}{|c|c|c|c|c|c|}
\hline Título y descripción del DDC \\
\hline 9- Fotografiando la Biosfera. Orientado a la creación de una fotografía panorámica sobre aspectos de geografía física, donde los estudiantes \\
son capaces de identificar y reconstruir características del paisaje y las definiciones que les corresponden. Esta fotografía se compartirá y
\end{tabular}
permitirá la discusión con un aula gemela de otro establecimiento educacional utilizando la plataforma Gigapan.

10- Un diaporama del siglo XX. Conducente a la creación de un diaporama que será publicado como video en el sitio Youtube. Este producto es elaborado a partir de una investigación sobre los Derechos Humanos en el siglo XX. Una vez publicado un video por grupo los trabajos son revisados por grupos gemelos que evalúan y comentan, generando una retroalimentación del proceso de aprendizaje. Durante la experiencia didáctica los estudiantes serán expuestos a diversas situaciones relacionadas con hechos relevantes de la historia mundial del siglo XX en donde confrontarán sus visiones y comunicarán sus ideas y reflexiones.

11- El blog de la cultura grecolatina. Trabajo de construcción de un blog integrando los productos textuales elaborados clase a clase hasta llegar a publicar la entrevista ficticia de un personaje griego o romano. En primer lugar hay un acercamiento y apropiación de contenido de la unidad a partir del conocimiento del legado político-cultural greco-romano y la elaboración de una actividad para lograr la apropiación de contenidos y conocimiento de la web 2.0. Luego, se hace una reflexión del propio trabajo y establecer intercambio con el aula gemela a partir de comentarios en los blog.

12- Un panoramio de nuestro mundo. Los estudiantes descubren, analizan y comparan distintos lugares geográficos, sus aspectos culturales y actividades económicas representativas apoyándose en un viaje virtual utilizando Panoramio. Cada grupo trabaja construyendo una descripción de lugares geográficos, primero extranjeros, luego locales, analizando a través de las fotografías aspectos del paisaje, vida urbana, cultura. El trabajo de los estudiantes se publica en Panoramio, permitiendo que sea accedido y comentado por otros estudiantes.

13- Creando una Monarquía Blog. Aborda el estudio de las monarquías absolutas ejercidas durante Europa entre los siglos XVI al XVIII mediante la construcción de un blog donde las y los estudiantes crearán una monarquía ficticia al estilo de un juego de roles que integre elementos caracterizadores a partir de los contenidos tratados. Los blog creados por los estudiantes serán compartidos y evaluados por los grupos de un aula gemela.

14- Fotografiando la Revolución Industrial. Comprende el estudio de las características de la Revolución Industrial, sus implicancias sociales, avances técnicos y tecnológicos, a través de equipos de investigación, sintetizarán y desplegarán la información a través de representaciones de cada tema fotografiadas y publicadas en GigaPan. Estas representaciones se compartirán con un aula gemela con el fin de generar la discusión en torno a los hechos estudiados y sus proyecciones con la sociedad contemporánea.

15- Nuestra revista de historia de Chile. Elaboración de una publicación virtual sobre las relaciones español e indígena durante los primeros años de la colonia, las cuales serán descritas, interpretadas y analizadas críticamente a partir de un texto de carácter periodístico-investigativo publicado en la web de revista digital Issuu; cuyo principal objetivo es abordar el contenido de persistencia y cultura indígena de forma crítica y constructiva.

16- Reconstruyendo nuestro patrimonio cultural. Organización de grupos colaborativos que realizan una investigación acerca del Patrimonio Cultural material e inmaterial que se haya en su localidad. Para esto, llevan a cabo entrevistas y salidas a terreno, a partir de las cuales los estudiantes reconocen el proceso de colonización en su región mediante la arquitectura, museos, crónicas y actores sociales presentes en la ciudad. La información es recopilada a partir de entrevistas, fotografías y lecturas que son sistematizadas en un video publicado en Youtube, en el cual los estudiantes exponen las dimensiones económica, social, geográfica y política. 


\section{DISCUSIÓN: CÓMO TRANSITAR HACIA UNA DIDÁCTICA 2.0}

Al margen de los alcances efectivos de instalación del proyecto la discusión que buscamos dice relación no tan sólo respecto del uso intensivo o variado de las TIC, sino más bien en función de cómo y para qué las usamos, por ello recalcamos que nuestra propuesta es fundamentalmente didáctica. De allí que acuñamos el concepto de didáctica 2.0 (Cárcamo, Scheihing y Cárdenas, 2013) para identificar este modo de organización que recoge para el hacer pedagógico, conductas habituales de los jóvenes en la Web 2.0: crear, publicar contenido, compartirlo y valorarlo (Crook 2012).

\subsection{Creación multimedial insertada curricularmente}

La propuesta persigue a partir de la creación de un producto (evidencia de aprendizaje) estimular el desarrollo del contenido disciplinar en la unidad respectiva, a través de procedimientos de búsqueda, selección y construcción, el cual es publicado en una Web Social. Igualmente, para Ivala, Gachago, Condy y Chigona (2013), la narrativas visuales resultan ser una síntesis de las temáticas investigadas por los estudiantes, en Kelluwen la creación multimedial es una alternativa pertinente en tanto los escolares están familiarizados con este tipo de mensajes y eso facilita enormemente los resultados del aprendizaje, sin embargo el paso necesario para poner la innovación al servicio de los objetivos educativos está en insertarla curricularmente. En muchos establecimientos la innovación con TIC, forma parte de actividades extraescolares, perdiendo así la oportunidad de renovar el aula.

Kelluwen incentiva al profesor a utilizar los mismos productos que elaboran los estudiantes como recursos para integrar información sobre los contenidos (específicamente conceptuales) que se deben alcanzar en la unidad o contenido curricular abordado.

\subsection{Valoración, encuentro y participación en las redes sociales}

En las redes sociales, los jóvenes tempranamente adquieren hábitos de valoración de contenidos escritos y visuales (Colás, González y de Pablos, 2013), el proyecto busca que los estudiantes valoren los productos o evidencias de aprendizaje que son compartidos en la plataforma de aprendizaje Kelluwen y en las herramientas de Web Social que cada DDC propone.

Desde esta perspectiva la utilización de las herramientas infocomunicacionales en el aprendizaje se orienta, naturalmente, a la incorporación de las expe- riencias previas en los DDC, ya que cada vez son más conocidas por los estudiantes las dinámicas de crear cuentas de usuario, editar perfiles, expresar opiniones en sitios web y valorar contenidos digitales. Así, pues, las acciones comunicativas mediadas digitalmente que ya son cotidianas para los jóvenes, son las utilizadas en la propuesta de Kelluwen para orientar los procesos de enseñanza-aprendizaje (Crook, 2012).

Con ello los estudiantes se organizan para elaborar un producto de carácter público, que adquiere visibilidad en la Web 2.0 y además esperan que este sea valorado y comentado por pares a través de la plataforma de aprendizaje 2.0 que ofrece Kelluwen. Así, por ejemplo, un video realizado por estudiantes de la ciudad de Valdivia (capital Región de los Ríos - Chile) puede ser visualizado y comentado por estudiantes de la localidad rural como Hualaihué, distante unos 300 kms., u otras. Finalmente tanto la publicación de las evidencias, como las valoraciones en red y las co-evaluaciones entre aulas gemelas resultan muy relevantes para conseguir procesos de autoregulación para los estudiantes en el sentido que Álvarez (2009) propone.

\subsection{El docente como innovador}

En acuerdo con Suarez (2013), el estudio del uso de TIC en contextos educativos debe ser atendido de modo multivariado. Así encontramos factores de orden contextuales y personales, orientados al profesorado y sus competencias digitales o informáticas. Por ello, es factible pensar que ofrecer propuestas de enseñanza para el aula escolar, que otorguen protagonismo al profesorado en el uso didáctico de las TIC tendrá una repercusión relevante en el aprendizaje de sus estudiantes.

Los DDC proponen un rol del docente como mediador y guía del proceso de enseñanza-aprendizaje. Si bien, en las planificaciones se puede observar un rol protagónico de los estudiantes, puesto que son ellos quienes se enfrentan al proceso de descubrimiento y construcción del conocimiento, debemos destacar el rol que ejerce cada profesor/a motivando y orientando las actividades que son propuestas, cambiando incluso sus propias concepciones respecto del efecto de las TIC (Albirini, 2006).

Por su parte, las actividades orientadas al trabajo colaborativo en el que cada estudiante se apropia de un rol activo comprometido con su equipo de trabajo y con la evidencia de aprendizaje que deben elaborar. Para Álvarez (2009) el comportamiento autorregulado refleja el compromiso del alumno con la tarea, 
su deseo de realizarla y, por tanto, compromete su motivación y voluntad. En este sentido, los estudiantes relacionan sus aprendizajes con los trabajos que evalúan estableciendo puntos de comparación, en la medida que las diferencias entre una evidencia y otra posibilitan la retroalimentación de los contenidos curriculares. Además, se facilita una instancia para realizar opiniones críticas basadas en los soportes audioescritovisuales o escritovisuales construidos por cada grupo de trabajo y el respeto a las creaciones del otro.

Con todo, Kelluwen se esfuerza en aportar a que las brechas de inserción cultural de la innovación se reduzcan a través de la incorporación de estrategias propias de redes sociales de Internet, generando una introducción dinámica en educandos motivados y profesores que se caracterizan por su proactividad. En el proceso de escalamiento de la innovación estos docentes se trasforman en agentes de cambio y de diseminación hacia sus pares (Pablos, Colás y González, 2010).

\section{CONCLUSIONES: LA NECESIDAD DE INSTALAR LA INNOVACIÓN CON TIC EN CONTEXTOS ESCOLARES}

Los DDC elaborados por Kelluwen son capaces de integrar las particularidades de los estudiantes a los que van dirigidos. Es así como matices propiciados por la edad, el nivel de enseñanza, la procedencia geográfica o socio-económica, los intereses de ocio y cultura como grupos musicales, películas, comunidades virtuales de interés, etc., pueden aportar a la creación de evidencias de aprendizaje más pertinentes y significativas para los estudiantes.

Como se ha evidenciado, no cabe duda que uno de los problemas más desafiantes para los sistemas educativos es institucionalizar en los colegios una cultura de innovación y cambio constante. La permeabilidad de la innovación resulta parcial o en muchos casos anecdótica y, por ello, cuesta revelar avances sustantivos en los aprendizajes (Haro de, 2009). No existen recetas mágicas para resolver esto, pero resulta necesario considerar que cualquier iniciativa que se proponga debe asumir un trabajo conjunto de todos los actores del proceso educativo, así como llevar a cabo implementaciones prácticas en los contextos reales de ejercicio docente. Ya no son suficientes iniciativas iluminadas que llegan a la escuela con soluciones inmediatistas, se requiere de una innovación arraigada en el proyecto educativo del centro escolar.

Insistimos en que el proyecto rescata, en primer lugar, que los jóvenes son activos y entusiastas usuarios de los servicios de la Web (Ricoy, Feliz y Sevillano,
2010) y, en segundo lugar, cada vez existe un mayor acceso a la tecnología en las escuelas (Tancer, 2007). Los profesores son conscientes de esto, pero no siempre encuentran herramientas formativas ni apoyos directivos para gestar la innovación pertinente.

El desconectado del mañana es como el analfabeto de hoy, pues el principal punto de desigualdad se hace tangible en el acceso, pero principalmente en el uso y control de los medios digitales, expresada hoy en la denominada convergencia digital (Arancibia, 2007). Asimismo, la nueva economía, afirma Castells (2001), se organiza en torno a redes globales de capital, gestión e información, cuyo acceso al conocimiento tecnológico constituye la base de la productividad y la competencia. En este aspecto, las funciones y procesos de lo que este autor denomina Sociedad Red, cada vez se estructuran más alrededor de tejidos sociales y materiales interconectados. En esta coyuntura, las TIC no sólo posibilitan la aparición de una nueva dimensión sociocultural, sino que, además, entregan la infraestructura para nuevos tipos de morfologías socioculturales a escala global. Kelluwen aborda esta problemática desde una mirada regional, asumiendo desde ya el uso del neologismo glocal para darle un sentido local a una propuesta que surge de un espacio y tiempo específico.

Los estudios sobre el aprendizaje con TIC expresan que toda incorporación a los procesos formativos escolares está mediada por el profesor, agente que, en última instancia, resuelve y define su uso (Pablos, et al., 2010; Sigalès, et al., 2009). Por tanto, no es sólo fundamental intervenir en el qué o cómo se aprende usando TIC, sino también qué se hace con ellas en el aula, qué hace al profesor tomar decisiones respecto de sus propuestas didácticas con uso de TIC, entendido esto como un fenómeno complejo (Phelps, Graham y Watts, 2011).

Por tanto, las pantallas digitales no pueden pasar a convertirse en pizarras donde únicamente se expone información a los estudiantes, reproduciendo modelos centrados en el profesor y la recepción pasiva por parte de los primeros. Más bien, se debe privilegiar el uso de las tecnologías para producir contenido, presentarlo y valorarlo, agregando valor al conocimiento que se construye en la sala de clases, utilizando recursos que en muchos casos los estudiantes ya portan, como son sus teléfonos móviles. En consecuencia, independiente de las TIC que se usen, lo fundamental es transitar hacia una didáctica 2.0 que promueva la colaboración propia de la red social, diseñada para estudiantes que son nativos de estos entornos, donde 
los fenómenos de interacción son instantáneos y las modalidades de comunicación cada vez más efímeras pero a la vez permanentes, pues todo queda en la red.

Kelluwen ha intentado responder a esta necesidad de acercar los modos de hacer de los jóvenes con las tareas que les solicitan en sus rutinas de educación formal, usando recursos tecnológicos variados, actuales y promoviendo que los profesores hagan de sus clases un acto cada vez más colaborativo.

\subsection{Dificultades y Limitaciones}

Al cerrar el presente artículo cabe señalar que pese a las bondades expuestas, el proyecto de Investigación, Desarrollo e Innovación Kelluwen hace también una lectura autocrítica de varios aspectos que dificultaron el trabajo en terreno y pueden servir de experiencia para otros equipos de investigación.

a) La innovación didáctica con TIC lucha fuertemente con la tradición. Aún en países que tienen una buena cobertura tecnológica como Chile y más de 15 años de masificación de la informática educativa, el profesor tiende a optar por prácticas tradicionales, sobre todo porque resultan economizadoras de tiempo en un contexto laboral con contratos que maximizan la presencia del maestro en la sala de clases y dejan escaso tiempo para planificar una innovación.

b) El contexto educativo chileno contempla la participación de todos los establecimientos en pruebas estandarizadas pertenecientes al Sistema Nacional de Medición de la Calidad de la Educación (SIMCE). Dichas evaluaciones determinan que en muchos establecimientos se establezcan dinámicas de entrenamiento que cierra las puertas a los esfuerzos de innovación.

c) El vínculo entre las universidades y los contextos educativos reales de enseñanza primaria y secundaria cuentan con escaza sinergia. Las instituciones universitarias se acercan a las escuelas, regularmente, con la finalidad de gestionar prácticas para los estudiantes de pedagogía, pero ese tipo de vínculo es insuficiente a la hora de implementar investigación aplicada. Se deben hacer grandes e intuitivos esfuerzos por institucionalizar convenios con los diferentes agentes del sistema administrativo que no garantizan con transparencia la ejecución de las experiencias de investigación, ni reconocen de alguna manera al profesor que desde su establecimiento se suma a las propuestas de innovación.

Con todas esas dificultades que limitaron el accionar del equipo de investigación, el proyecto es capaz de exhibir resultados concretos y positivos. Sin duda, gran parte de estos éxitos se deben a la voluntad puesta por los profesores que en tres años acogieron la innovación y aportaron constantemente para su mejora.

\section{AGRADECIMIENTOS}

Los autores agradecen el financiamiento del Fondo de Fomento al Desarrollo Científico y Tecnológico (FONDEF), de la Comisión Nacional de Investigación Científica y Tecnológica de Chile (CONICYT) y el Patrocinio de la Dirección de Investigación y Desarrollo (DID) de la Universidad Austral de Chile. 
1 Kelluwen es un vocablo del mapudungún (lengua mapuche, pueblo originario de Chile) que significa "trabajar entre todos", aunque para esta iniciativa cobra un significado mayor: nuestro mensaje es "trabajar y aprender colaborativamente". Kelluwen con financiamiento de FONDEF-CONICYT (código D80i-1074), construye paulatina y colaborativamente una comunidad educativa de escolares, profesores e investigadores abocada a trabajar con/en las escuelas y liceos vulnerables del Sur Austral de Chile.

\section{BIBLIOGRAFÍA}

Álvarez Valdivia, I. M. (2009). "Evaluar para contribuir a la autorregulación del aprendizaje". Electronic Journal of Research in Educational Psychology, 19, pp. 1007-1030.

Albirini, A. (2006). "Teachers' attitudes towards information and communication technologies: The case of Syrian EFL teachers". Computers \& Education, 47, pp. 373-398. DOI: http://dx.doi. org/10.1016/j.compedu.2004.10.013

Arancibia Herrera, M. (2000). “La Red Enlaces en Chile. Incorporación de la Informática a las Tareas Pedagógicas: una Historia que Alumbra un Promisorio Futuro". Perspectiva Educacional, vol 35-36, pp. 133-151.

Arancibia Herrera, M. (2007). "Uso de medios y TIC en escuelas ¿cómo disminuir la brecha digital?". Visiones de la Educación, vol 12, pp. 17-30.

Arancibia Herrera, M. y Contreras Contreras, P. (2013). "¿Qué debemos saber antes de hacer la minga kelluwen?". Estudios sobre el uso de TIC en el aula. En: Cárcamo, Scheihing y Cárdenas, Didáctica 2.0: La web social en el aula (pp. 21-36). Valdivia: Ediciones Kelluwen.

Aubert Simon, A., Flecha Fernandez A, García Yeste C, Flecha García R. y Racionero Plaza S. (2011). Aprendizaje Dialógico en la Sociedad de la Información. Barcelona: Hipatia.

Bartolomé Pina, A. (2004). "Blended Learning. Conceptos básicos". Píxel-Bit. Revista de Medios y Educación, vol 23, pp. 7-20.
Beyer Burgos, H. (2000). "Entre la autonomía y la intervención: las reformas de la educación en Chile". En: Larraín, F. y Vergara, R. (Eds.), La transformación económica de Chile (pp. 643-708). Santiago: CEP.

Bonder, G. (2008). “Juventud, género \& TIC: imaginarios en la construcción de la sociedad de la información en América Latina". ARBOR Ciencia, Pensamiento y Cultura CLXXXIV, pp. 917 - 934. DOI:10.3989/arbor.2008.i733.234

Castells Oliván, M. (2001). La galaxia Internet. Barcelona: Areté.

Cárcamo Ulloa, L., Scheihing García, E. \& Cárdenas Neira, C. (eds.) (2013). Didáctica 2.0: la web social en el aula. Valdivia: Ediciones Kelluwen.

Cebrian Herrero, M. (2009). “Comunicación interactiva en los cibermedios". Comunicar, 33, pp.15-24.

Colás Bravo, P., González Ramirez, T. \& De Pablos Pons, J. (2013). "Juventud y redes sociales: Motivaciones y usos preferentes". Comunicar, 40(XX); 15 23. DOI: http://dx.doi.org/10.3916/ C40-2013-02-01

Coll Salvador, César. (2005) “Lectura y analfabetismo en la Sociedad de la Información". UOCPapers № 1. Recuperado de: http://www.uoc.edu/uocpapers/1/dt/ esp/coll.pdf

Chinn Menzie D. y Fairlie W, R. (2010). "ICT Use In The Developing World: An Analysis Of Differences In Computer And Internet Penetrationroie". Review of International Economics, vol 18, no1, pp. 153-167. DOI: 10.1111/j.14679396.2009.00861.x

Cloutier, J. (2001). Petit traité de communication. Emerec à l'heu re des technologies numériques. Montréal: Carte Blanche.

Cox Donoso, C. (2005). "Las políticas educacionales de Chile en las dos últimas décadas del siglo XX". En: Cox, C. (Ed.), Políticas educacionales en el cambio de siglo. La reforma del sistema escolar de Chile (pp. 19-113). Santiago: Editorial Universitaria.

Crook, Ch. R. (2012). "The 'digital native' in context: tensions associated with importing Web 2.0 practices into the school setting". Oxford Review of Education, vol 38, no 1, pp 63-80. DOI: http://dx.doi.org/10.1080/03054985.2 011.577946

De Pablos Pons, J., Colás Bravo, P., González Ramírez, T. (2010). "Factores facilitadores de la innovación con TIC en los centros escolares. Un análisis comparativo entre diferentes políticas educativas autonómicas". Revista de Educación, 352, pp. 23-51.

García-Valcárcel, A. y Tejedor Tejedor, F. J. (2010). “Evaluación de los procesos de innovación escolar basados en el uso de las TIC desarrollados en la Comunidad de Castilla y León". Revista de Educación, vol 352, pp. 125-147.

Gardner, H. (1993). La mente no escolarizada. Cómo piensan los niños y cómo deberían enseñar las escuelas. Barcelona: Paidós. 
Haro de, J. J. (2009). "Algunas experiencias de innovación educativa". ARBOR Ciencia, Pensamiento y Cultura CLXXXV pp. 71 - 92. DOI:10.3989/arbor.2009. extran1207

Hepp Kushel, P. E (2003). “ENLACES: El Programa de Informática Educativa de la Reforma Educacional Chilena". En: Cox, C. (Ed.), Políticas educacionales en el cambio de siglo. La reforma del sistema escolar de Chile (pp. 419-451). Santiago: Editorial Universitaria.

Hillis, P. y Munro, B. (2005). "ICT in History Education Scotland and Europe". Social Science Computer Review, vol 23, pp. 190-205. doi: 10.1177/0894439304273268

Ivala, E. , Gachago, D. , Condy, J. \& Chigona, A. (2013). "Enhancing Student Engagement with Their Studies: A Digital Storytelling Approach". Creative Education, 4, 82-89. doi: 10.4236/ ce.2013.410A012.

Meier, E. (2005). "Situating Technology professional development in urban schools". Educational Computing Research, vol 32, n4, pp. 395-407.

MINEDUC. (2003). Marco para la Buena Enseñanza. Santiago: MINEDUC. Recuperado de: http://www.educarchile.cl/ ech/pro/app/detalle?ID=139671
Monereo Font, C. (2005). Internet y competencias básicas. Aprender a colaborar, a comunicarse, a participar, a aprender. Barcelona: Grao.

Palacheewa, P., Suwannatthachote, P. \& Nilsook, P. (2012). "Critical Issues To Be Concern In Selecting Tools For Teaching High School Computer Project Lesson Using Computer-Supported Collaborative Learning". Creative Education, vol 3, pp.11-14. doi: 10.4236/ce.2012.38B003

Phelps, R., Graham, A. y Watts, T. (2011). "Acknowledging The Complexity And Diversity Of Historical And Cultural ICT Professional Learning Practices In Schools". Asia-Pacific Journal of Teacher Education, vol 39, no 1, pp. 47-63. doi: 10.1080/1359866X.2010.541601

Prain, V. y Hand, B. (2003). "Using new technology for learning: a case study of a whole school approach". Journal of research on technology in education, vol $35, \mathrm{n}$ ㄴ, pp. 441-458.

Prieto Castillo, D. (1996). Palabras e imágenes para la comunicación impresa. Quito: UCLAP.

Ricoy Lorenzo, M C .; Feliz Murias, S. y Sevillano García, M L. (2010). “Competencias para la utilización de las herramientas digitales en la sociedad de la información". Educación XX1, vol 13.no 1, pp. 199-219
Sigalès Conde, C., Mominó De La Iglesia, J M., Meneses, J. y Badia Garganté, A. (2009). La integración de internet en la educación escolar española. Barcelona: Ariel.

SITES (2008). Resultados nacionales SITES 2006. Santiago: MINEDUC-Enlaces. Recuperado de: http://goo.gl/Jj2ozd

Staples, A., Pugach, M. y Himes, D. (2005)."Rethinking the technology integration Challenge: Cases from three urban elementary schools". Journal of research in technology in education, vol 37 , no 3, pp. 285-311.

Suárez Rodríguez, J.; Almerich, G.; Gargallo López, B. y Aliaga, F. (2013). "Las competencias del profesorado en TIC: estructura básica". Educación XX1, vol 16, №.1, pp. 39-62.

Tancer, B. (2007). “Facebook: More Popular Than Porn. Time Magazine". Recuperado en http://content.time.com/time/business/article/0,8599,1678586,00.html

Zhao, Y. y Frank, K. (2006). "Factors affecting Technology uses in schools: An ecological Perspective". American Educational Research Journal, vol 40, no 4, pp. 807840. doi: 10.3102/00028312040004807 